\title{
New inequalities on eigenvalues of the Hadamard product and the Fan product of matrices
}

\author{
Qian-Ping Guo*, Hou-Biao Li* and Ming-Yan Song
}

\section{"Correspondence:}

guogianpinglei@163.com;

lihoubia00189@163.com

School of Mathematical Sciences,

University of Electronic Science and

Technology of China, Chengdu,

611731, P.R. China

\begin{abstract}
In the paper, some new upper bounds for the spectral radius of the Hadamard product of nonnegative matrices, and the low bounds for the minimum eigenvalue of the Fan product of nonsingular $M$-matrices are given. These new bounds improve existing results, and the estimating formulas are easier to calculate since they only depend on the entries of matrices. Finally, some examples are also given to show that the bounds are better than some previous results.
\end{abstract}

MSC: 65F10; 65F15; 65F50

Keywords: Hadamard product; nonnegative matrices; spectral radius; Fan product; M-matrix; minimum eigenvalue

\section{Introduction}

In this paper, for a positive integer $n, N$ denotes the set $\{1,2, \ldots, n\} . \mathbb{R}^{n \times n}\left(\mathbb{C}^{n \times n}\right)$ denotes the set of all $n \times n$ real (complex) matrices. Let $A=\left(a_{i j}\right)$ and $B=\left(b_{i j}\right)$ be two real $n \times n$ matrices. We write $A \geq B(A>B)$ if $a_{i j} \geq b_{i j}\left(a_{i j}>b_{i j}\right)$ for all $i, j \in N$. If $A \geq 0(A>0)$, we say $A$ is a nonnegative (positive) matrix. The spectral radius of $A$ is denoted by $\rho(A)$. If $A$ is a nonnegative matrix, the Perron-Frobenius theorem guarantees that $\rho(A) \in \sigma(A)$, where $\sigma(A)$ is the set of all eigenvalues of $A$ throughout this paper (see [1]).

For $n \geq 2$, an $n \times n$ matrix $A$ is said to be reducible if there exists a permutation matrix $P$ such that

$$
P^{T} A P=\left(\begin{array}{ll}
B & C \\
0 & D
\end{array}\right),
$$

where $B$ and $D$ are square matrices of order at least one. If no such permutation matrix exists, then $A$ is called irreducible. If $A$ is a $1 \times 1$ complex matrix, then $A$ is irreducible if and only if its single entry is nonzero (see [2]).

According to Ref. [3], a matrix $A$ is called an $M$-matrix if there exists an $n \times n$ nonnegative real matrix $P$ and a nonnegative real number $\alpha$ such that $A=\alpha I-P$ and $\alpha \geq \rho(P)$, where $I$ is the identity matrix. Moreover, if $\alpha>\rho(P), A$ is called a nonsingular $M$-matrix; if $\alpha=\rho(P)$, we call $A$ a singular $M$-matrix.

In addition, a matrix $A=\left(a_{i j}\right) \in \mathbb{R}^{n \times n}$ is called $Z$-matrix if all of it off-diagonal entries are negative and denoted by $A \in Z_{n}$. For convenience, the following simple facts are needed

\section{算 Springer}

(c) 2013 Guo et al.; licensee Springer. This is an Open Access article distributed under the terms of the Creative Commons Attribution License (http://creativecommons.org/licenses/by/2.0), which permits unrestricted use, distribution, and reproduction in any medium, provided the original work is properly cited. 
(see Problems 16, 19 and 28 in Section 2.5 of [3]), where $\tau(A) \triangleq \min \{\lambda \mid \lambda \in \sigma(A)\}$, and $M_{n}$ is denoted by the set of all nonsingular $M$-matrices (see [1]):

1. $\tau(A) \in \sigma(A)$;

2. If $A, B \in M_{n}$ and $A \geq B$, then $\tau(A) \geq \tau(B)$;

3. If $A \in M_{n}$, then $\rho\left(A^{-1}\right)$ is the Perron eigenvalue of the nonnegative matrix $A^{-1}$, and $\tau(A)=\frac{1}{\rho\left(A^{-1}\right)}$ is a positive real eigenvalue of $A$.

Let $A$ be an irreducible nonnegative matrix. It is well known that there exist positive vectors $u$ and $v$ such that $A u=\rho(A) u$ and $v^{T} A=\rho(A) v^{T}$, where $u$ and $v$ are right and left Perron eigenvectors of $A$, respectively.

The Hadamard product of $A=\left(a_{i j}\right) \in \mathbb{C}^{n \times n}$ and $B=\left(b_{i j}\right) \in \mathbb{C}^{n \times n}$ is defined by $A \circ B=$ $\left(a_{i j} b_{i j}\right) \in \mathbb{C}^{n \times n}$.

For two real matrices $A, B \in M_{n}$, the Fan product of $A$ and $B$ is denoted by $A \star B=C=$ $\left[c_{i j}\right] \in M_{n}$ and is defined by

$$
c_{i j}= \begin{cases}-a_{i j} b_{i j} & \text { if } i \neq j, \\ a_{i i} b_{i i} & \text { if } i=j .\end{cases}
$$

Obviously, if $A, B \in M_{n}$, then $A \star B$ is also an $M$-matrix (see [2]).

We define

$$
\begin{aligned}
& R_{i}=\sum_{k \neq i}\left|a_{i k}\right|, \quad d_{i}=\frac{R_{i}}{\left|a_{i i}\right|}, \quad i \in N ; \\
& r_{l i}=\frac{\left|a_{l i}\right|}{\left|a_{l l}\right|-\sum_{k \neq l, i}\left|a_{l k}\right|}, \quad l \neq i ; \quad r_{i}=\max _{l \neq i}\left\{r_{l i}\right\}, \quad i \in N ; \\
& s_{j i}=\left|a_{j i}\right| m_{j}, \quad m_{j}=\left\{\begin{array}{ll}
r_{j} & \text { if } r_{j} \neq 0, \\
1 & \text { if } r_{j}=0 ;
\end{array} s_{i}=\max _{j \neq i}\left\{s_{j i}\right\}, \quad i, j \in N,\right.
\end{aligned}
$$

throughout the paper.

The paper is organized as follows. Firstly, for two nonnegative matrices $A$ and $B$, we exhibit some new upper bounds for $\rho(A \circ B)$ in Section 2. In Section 3, some new lower bounds for $\tau(A \star B)$ of $M$-matrices are presented. Finally, some examples are given to illustrate our results.

\section{Some upper bounds for the spectral radius of the Hadamard product of two nonnegative matrices}

Firstly, in ([3], p.358), there is a simple estimate for $\rho(A \circ B)$ : if $A, B \in \mathbb{R}^{n \times n}, A \geq 0$, and $B \geq 0$, then

$$
\rho(A \circ B) \leq \rho(A) \rho(B) .
$$

Recently, Fang [4] gave an upper bound for $\rho(A \circ B)$, that is,

$$
\rho(A \circ B) \leq \max _{1 \leq i \leq n}\left\{2 a_{i i} b_{i i}+\rho(A) \rho(B)-b_{i i} \rho(A)-a_{i i} \rho(B)\right\},
$$

which is smaller than the bound $\rho(A) \rho(B)$ in ([3], p.358). 
Liu and Chen [2] improved (2.2) and gave the following result:

$$
\begin{aligned}
\rho(A \circ B) \leq & \max _{1 \leq i \leq n} \frac{1}{2}\left\{a_{i i} b_{i i}+a_{j j} b_{j j}+\left[\left(a_{i i} b_{i i}-a_{j j} b_{j j}\right)^{2}\right.\right. \\
& \left.\left.+4\left(\rho(A)-a_{i i}\right)\left(\rho(B)-b_{i i}\right)\left(\rho(A)-a_{j j}\right)\left(\rho(B)-b_{j j}\right)\right]^{\frac{1}{2}}\right\} .
\end{aligned}
$$

Recently, some elaborate new bounds were also presented in [5], which in some cases give better estimates for the spectral radius of the Hadamard product of two nonnegative matrices.

In this section, based on the idea of [5], we present some new upper bounds on $\rho(A \circ B)$ for nonnegative matrices $A$ and $B$ which improve the above results. The new estimating formulae also only depend on the entries of matrices $A$ and $B$.

Lemma 2.1 [6] Let $A=\left(a_{i j}\right)$ be an arbitrary complex matrix, and let $x_{1}, x_{2}, \ldots, x_{n}$ be positive real numbers, then all the eigenvalues of $A$ lie in the region

$$
G(A)=\bigcup\left\{z \in \mathbb{C}:\left|z-a_{i i}\right| \leq x_{i} \sum_{j \neq i} \frac{1}{x_{j}}\left|a_{j i}\right|, i \in N\right\} .
$$

Lemma 2.2 [7] Let $A=\left(a_{i j}\right) \in \mathbb{C}^{n \times n}$, and let $x_{1}, x_{2}, \ldots, x_{n}$ be positive real numbers, then all the eigenvalues of $A$ lie in the region

$$
B(A)=\bigcup_{i, j=1 ; i \neq j}^{n}\left\{z \in \mathbb{C}:\left|z-a_{i i}\right|\left|z-a_{i j}\right| \leq\left(x_{i} \sum_{k \neq i} \frac{1}{x_{k}}\left|a_{k i}\right|\right)\left(x_{j} \sum_{k \neq j} \frac{1}{x_{k}}\left|a_{k j}\right|\right)\right\} .
$$

Next, we present a new estimating formula of the upper bounds of $\rho(A \circ B)$ which is easier to calculate.

Theorem 2.1 If $A=\left(a_{i j}\right)$ and $B=\left(b_{i j}\right)$ are nonnegative matrices, then

$$
\rho(A \circ B) \leq \max _{1 \leq i \leq n}\left\{a_{i i} b_{i i}+s_{i} \sum_{j \neq i} \frac{b_{j i}}{m_{j}}\right\}
$$

Proof It is evident that inequality (2.6) holds with equality for $n=1$. Therefore, we assume that $n \geq 2$ and give two cases to prove this problem.

Case 1. Suppose that $C=A \circ B$ is irreducible. Obviously $A$ and $B$ are also irreducible. By Lemma 2.1, there exists $i_{0}\left(1 \leq i_{0} \leq n\right)$ such that

$$
\left|\rho(A \circ B)-a_{i_{0} i_{0}} b_{i_{0} i_{0}}\right| \leq s_{i_{0}} \sum_{k \neq i_{0}} \frac{a_{k i_{0}} b_{k i_{0}}}{s_{k}},
$$

i.e.,

$$
\begin{aligned}
\rho(A \circ B) & \leq a_{i_{0} i_{0}} b_{i_{0} i_{0}}+s_{i_{0}} \sum_{k \neq i_{0}} \frac{a_{k i_{0}} b_{k i_{0}}}{s_{k}} \\
& \leq a_{i_{0} i_{0}} b_{i_{0} i_{0}}+s_{i_{0}} \sum_{k \neq i_{0}} \frac{a_{k i_{0}} b_{k i_{0}}}{a_{k i_{0}} m_{k}}
\end{aligned}
$$




$$
\begin{aligned}
& =a_{i_{0} i_{0}} b_{i_{0} i_{0}}+s_{i_{0}} \sum_{k \neq i_{0}} \frac{b_{k i_{0}}}{m_{k}} \\
& \leq \max _{i}\left\{a_{i i} b_{i i}+s_{i} \sum_{k \neq i} \frac{b_{k i}}{m_{k}}\right\} .
\end{aligned}
$$

Thus, we have that

$$
\rho(A \circ B) \leq \max _{i}\left\{a_{i i} b_{i i}+s_{i} \sum_{k \neq i} \frac{b_{k i}}{m_{k}}\right\}
$$

So, conclusion (2.6) holds.

Case 2. If $C=A \circ B$ is reducible. We may denote by $P=\left(p_{i j}\right)$ the $n \times n$ permutation matrix $\left(p_{i j}\right)$ with

$$
p_{12}=p_{23}=\cdots=p_{n-1, n}=p_{n, 1}=1,
$$

the remaining $p_{i j}$ being zero, then both $A+\varepsilon P$ and $B+\varepsilon P$ are nonnegative irreducible matrices for any sufficiently small positive real number $\varepsilon$. Now we substitute $A+\varepsilon P$ and $B+\varepsilon P$ for $A$ and $B$, respectively, in the previous Case 1 , and then letting $\varepsilon \rightarrow 0$, the result (2.6) follows by continuity.

Theorem 2.2 If $A=\left(a_{i j}\right)$ and $B=\left(b_{i j}\right)$ are nonnegative matrices, then

$$
\begin{aligned}
\rho(A \circ B) \leq & \max _{i \neq j} \frac{1}{2}\left\{a_{i i} b_{i i}+a_{i j} b_{j j}+\left[\left(a_{i i} b_{i i}-a_{j j} b_{j j}\right)^{2}\right.\right. \\
& \left.\left.+4 s_{i} s_{j}\left(\sum_{k \neq i} \frac{b_{k i}}{m_{k}}\right)\left(\sum_{l \neq j} \frac{b_{l j}}{m_{l}}\right)\right]^{\frac{1}{2}}\right\} .
\end{aligned}
$$

Proof Similarly, inequality (2.7) holds with equality for $n=1$. Therefore, we assume that $n \geq 2$ and give two cases to prove this problem.

Case 1. Suppose that $C=A \circ B$ is irreducible. Obviously, $A$ and $B$ are also irreducible. By Lemma 2.2 , there exists a pair $(i, j)$ of positive integers with $i \neq j(1 \leq i, j \leq n)$ such that

$$
\begin{aligned}
\left|\rho(A \circ B)-a_{i i} b_{i i}\right|\left|\rho(A \circ B)-a_{j j} b_{j j}\right| & \leq\left(s_{i} \sum_{k \neq i} \frac{a_{k i} b_{k i}}{s_{k}}\right)\left(s_{j} \sum_{l \neq j} \frac{a_{l j} b_{l j}}{s_{l}}\right) \\
& \leq\left(s_{i} \sum_{k \neq i} \frac{a_{k i} b_{k i}}{a_{k i} m_{k}}\right)\left(s_{j} \sum_{l \neq j} \frac{a_{l j} b_{l j}}{a_{l j} m_{l}}\right) \\
& =\left(s_{i} \sum_{k \neq i} \frac{b_{k i}}{m_{k}}\right)\left(s_{j} \sum_{l \neq j} \frac{b_{l j}}{m_{l}}\right) .
\end{aligned}
$$

From inequality (2.8) and $\rho(A \circ B) \geq a_{i i} b_{i i}$ (see [8]), for any $i \in N$, we have

$$
\left(\rho(A \circ B)-a_{i i} b_{i i}\right)\left(\rho(A \circ B)-a_{j j} b_{j j}\right) \leq\left(s_{i} \sum_{k \neq i} \frac{b_{k i}}{m_{k}}\right)\left(s_{j} \sum_{l \neq j} \frac{b_{l j}}{m_{l}}\right) .
$$


Thus, by solving quadratic inequality (2.9), we have that

$$
\begin{aligned}
\rho(A \circ B) & \leq \frac{1}{2}\left\{a_{i i} b_{i i}+a_{j j} b_{j j}+\left[\left(a_{i i} b_{i i}-a_{j j} b_{j j}\right)^{2}+4 s_{i} s_{j}\left(\sum_{k \neq i} \frac{b_{k i}}{m_{k}}\right)\left(\sum_{l \neq j} \frac{b_{l j}}{m_{l}}\right)\right]^{\frac{1}{2}}\right\} \\
& \leq \max _{i \neq j} \frac{1}{2}\left\{a_{i i} b_{i i}+a_{j j} b_{j j}+\left[\left(a_{i i} b_{i i}-a_{j j} b_{j j}\right)^{2}+4 s_{i} s_{j}\left(\sum_{k \neq i} \frac{b_{k i}}{m_{k}}\right)\left(\sum_{l \neq j} \frac{b_{l j}}{m_{l}}\right)\right]^{\frac{1}{2}}\right\},
\end{aligned}
$$

i.e., conclusion (2.7) holds.

Case 2. If $C=A \circ B$ is reducible. We may denote by $P=\left(p_{i j}\right)$ the $n \times n$ permutation matrix $\left(p_{i j}\right)$ with

$$
p_{12}=p_{23}=\cdots=p_{n-1, n}=p_{n, 1}=1
$$

the remaining $p_{i j}$ being zero, then both $A+\varepsilon P$ and $B+\varepsilon P$ are nonnegative irreducible matrices for any sufficiently small positive real number $\varepsilon$. Now we substitute $A+\varepsilon P$ and $B+\varepsilon P$ for $A$ and $B$, respectively, in the previous Case 1 , and then letting $\varepsilon \rightarrow 0$, the result (2.7) follows by continuity.

Remark 2.1 Next, we give a comparison between inequality (2.6) and inequality (2.7). Without loss of generality, for $i \neq j$, we assume that

$$
a_{i i} b_{i i}-s_{i} \sum_{k \neq i} \frac{b_{k i}}{m_{k}} \leq a_{j j} b_{j j}-s_{j} \sum_{l \neq j} \frac{b_{l j}}{m_{l}} .
$$

Thus, we can rewrite (2.10) as

$$
s_{j} \sum_{l \neq j} \frac{b_{l j}}{m_{l}} \leq a_{j j} b_{j j}-a_{i i} b_{i i}+s_{i} \sum_{k \neq i} \frac{b_{k i}}{m_{k}} .
$$

From (2.11), we have that

$$
\begin{aligned}
& \left(a_{i i} b_{i i}-a_{j j} b_{j j}\right)^{2}+4\left(s_{i} \sum_{k \neq i} \frac{b_{k i}}{m_{k}}\right)\left(s_{j} \sum_{l \neq j} \frac{b_{l j}}{m_{l}}\right) \\
& \leq\left(a_{i i} b_{i i}-a_{j j} b_{j j}\right)^{2}+4 s_{i} \sum_{k \neq i} \frac{b_{k i}}{m_{k}}\left(a_{j j} b_{j j}-a_{i i} b_{i i}+s_{i} \sum_{k \neq i} \frac{b_{k i}}{m_{k}}\right) \\
& \leq\left(a_{i i} b_{i i}-a_{j j} b_{j j}\right)^{2}+4 s_{i} \sum_{k \neq i} \frac{b_{k i}}{m_{k}}\left(a_{j j} b_{j j}-a_{i i} b_{i i}\right)+4\left(s_{i} \sum_{k \neq i} \frac{b_{k i}}{m_{k}}\right)^{2} \\
& \quad=\left(a_{j j} b_{j j}-a_{i i} b_{i i}+2 s_{i} \sum_{k \neq i} \frac{b_{k i}}{m_{k}}\right)^{2} .
\end{aligned}
$$

Thus, from (2.7) and the above inequality, we can obtain

$$
\begin{aligned}
\rho(A \circ B) \leq & \max _{i \neq j} \frac{1}{2}\left\{a_{i i} b_{i i}+a_{j j} b_{j j}\right. \\
& \left.+\left[\left(a_{i i} b_{i i}-a_{j j} b_{j j}\right)^{2}+4 s_{i} s_{j}\left(\sum_{k \neq i} \frac{b_{k i}}{m_{k}}\right)\left(\sum_{l \neq j} \frac{\left|b_{l j}\right|}{m_{l}}\right)\right]^{\frac{1}{2}}\right\}
\end{aligned}
$$




$$
\begin{aligned}
& \leq \max _{i \neq j} \frac{1}{2}\left\{a_{i i} b_{i i}+a_{j j} b_{j j}+a_{j j} b_{j j}-a_{i i} b_{i i}+2 s_{i} \sum_{k \neq i} \frac{b_{k i}}{m_{k}}\right\} \\
& \leq \max _{1 \leq i \leq n}\left\{a_{i i} b_{i i}+s_{i} \sum_{k \neq i} \frac{b_{k i}}{m_{k}}\right\} .
\end{aligned}
$$

Hence, the bound in (2.7) is sharper than the bound in (2.6).

Example 2.1 [1] Let

$$
A=\left(a_{i j}\right)=\left(\begin{array}{cccc}
4 & 1 & 1 & 1 \\
2 & 5 & 1 & 1 \\
0 & 2 & 4 & 1 \\
1 & 1 & 1 & 4
\end{array}\right), \quad B=\left(b_{i j}\right)=\left(\begin{array}{cccc}
1 & 1 & 0 & 0 \\
1 & 3 & 2 & 0 \\
0 & 1 & 4 & 3 \\
0 & 0 & 1 & 5
\end{array}\right)
$$

If we apply (2.1), we have

$$
\rho(A \circ B) \leq \rho(A) \rho(B)=50.1274 .
$$

If we apply (2.2), we have

$$
\rho(A \circ B) \leq \max _{1 \leq i \leq n}\left\{2 a_{i i} b_{i i}+\rho(A) \rho(B)-a_{i i} \rho(B)-b_{i i} \rho(A)\right\}=25.5364 .
$$

If we apply (2.3), we have

$$
\begin{aligned}
\rho(A \circ B) \leq & \max _{1 \leq i \leq n} \frac{1}{2}\left\{a_{i i} b_{i i}+a_{j j} b_{j j}+\left[\left(a_{i i} b_{i i}-a_{j j} b_{j j}\right)^{2}\right.\right. \\
& \left.\left.+4\left(\rho(A)-a_{i i}\right)\left(\rho(B)-b_{i i}\right)\left(\rho(A)-a_{j j}\right)\left(\rho(B)-b_{j j}\right)\right]^{\frac{1}{2}}\right\} \\
= & 25.3644 .
\end{aligned}
$$

If we apply Theorem 2.1, we get

$$
\rho(A \circ B) \leq \max _{1 \leq i \leq n}\left\{a_{i i} b_{i i}+s_{i} \sum_{j \neq i} \frac{b_{j i}}{m_{j}}\right\}=24 .
$$

If we apply Theorem 2.2, we obtain that

$$
\begin{aligned}
\rho(A \circ B) & \leq \max _{i \neq j} \frac{1}{2}\left\{a_{i i} b_{i i}+a_{i j} b_{i j}+\left[\left(a_{i i} b_{i i}-a_{j j} b_{j j}\right)^{2}+4 s_{i} s_{j} \sum_{k \neq i} \frac{b_{k i}}{m_{k}} \sum_{l \neq j} \frac{b_{l j}}{m_{l}}\right]^{\frac{1}{2}}\right\} \\
& =22.1633 .
\end{aligned}
$$

In fact, $\rho(A \circ B)=20.7439$. The example shows that the bounds in Theorem 2.1 and Theorem 2.2 are better than the existing bounds. 


\section{Inequalities for the Fan product of two $M$-matrices}

Firstly, let us recall some results. It is known (p.359, [3]) that the following classical result is given. If $A, B \in \mathbb{R}^{n \times n}$ are $M$-matrices, then

$$
\tau(A \star B) \geq \tau(A) \tau(B)
$$

In 2007, Fang improved (3.1) in Remark 3 of Ref. [4] and gave a new lower bound for $\tau(A \star B)$, that is,

$$
\tau(A \star B) \geq \min _{1 \leq i \leq n}\left\{b_{i i} \tau(A)+a_{i i} \tau(B)-\tau(A) \tau(B)\right\} .
$$

Subsequently, Liu and Chen [2] gave a sharper bound than (3.2), i.e.,

$$
\begin{aligned}
\tau(A \star B) \geq & \max _{1 \leq i \leq n} \frac{1}{2}\left\{a_{i i} b_{i i}+a_{j j} b_{j j}-\left[\left(a_{i i} b_{i i}-a_{j j} b_{j j}\right)^{2}\right.\right. \\
& \left.\left.+4\left(a_{i i}-\tau(A)\right)\left(b_{i i}-\tau(B)\right)\left(a_{j j}-\tau(A)\right)\left(b_{j j}-\tau(B)\right)\right]^{\frac{1}{2}}\right\} .
\end{aligned}
$$

Next, we exhibit a new lower bound on the minimum eigenvalue $\tau(A \star B)$ of the Fan product of nonsingular $M$-matrices.

Theorem 3.1 If $A=\left(a_{i j}\right)$ and $B=\left(b_{i j}\right)$ are nonsingular $M$-matrices, then

$$
\tau(A \star B) \geq \min _{1 \leq i \leq n}\left\{a_{i i} b_{i i}-s_{i} \sum_{j \neq i} \frac{\left|b_{j i}\right|}{m_{j}}\right\}
$$

Proof It is evident that inequality (3.4) holds with equality for $n=1$. Therefore, we assume that $n \geq 2$ and give two cases to prove this problem.

Case 1. Suppose that $C=A \star B$ is irreducible. Obviously, $A$ and $B$ are also irreducible. By Lemma 2.1, there exists $i(1 \leq i \leq n)$ such that

$$
\left|\tau(A \star B)-a_{i i} b_{i i}\right| \leq s_{i} \sum_{k \neq i} \frac{\left|a_{k i} b_{k i}\right|}{s_{k}} \leq s_{i} \sum_{k \neq i} \frac{\left|a_{k i} b_{k i}\right|}{\left|a_{k i}\right| m_{k}}=s_{i} \sum_{k \neq i} \frac{\left|b_{k i}\right|}{m_{k}}
$$

From inequality (3.5) and $0 \leq \tau(A \star B) \leq a_{i i} b_{i i}$ (see [8]), for any $i \in N$, we have

$$
a_{i i} b_{i i}-\tau(A \star B) \leq s_{i} \sum_{k \neq i} \frac{\left|b_{k i}\right|}{m_{k}}
$$

Thus, we can obtain that

$$
\tau(A \star B) \geq \min _{1 \leq i \leq n}\left\{a_{i i} b_{i i}-s_{i} \sum_{k \neq i} \frac{\left|b_{k i}\right|}{m_{k}}\right\}
$$

i.e., the conclusion (3.4) holds.

Case 2. If $C=A \star B$ is reducible. It is well known that a matrix in $Z_{n}$ is a nonsingular $M$-matrix if and only if all its leading principal minors are positive (see Condition (E17) of 
Theorem 6.2 .3 of [8]). If we denote by $P=\left(p_{i j}\right)$ the $n \times n$ permutation matrix $\left(p_{i j}\right)$ with

$$
p_{12}=p_{23}=\cdots=p_{n-1, n}=p_{n, 1}=1
$$

the remaining $p_{i j}$ being zero, then both $A-\varepsilon P$ and $B-\varepsilon P$ are irreducible nonsingular $M$-matrices for any sufficiently small positive real number $\varepsilon$ such that all the leading principal minors of both $A-\varepsilon P$ and $B-\varepsilon P$ are positive. Now we substitute $A-\varepsilon P$ and $B-\varepsilon P$ for $A$ and $B$, respectively, in the previous Case 1 , and then letting $\varepsilon \rightarrow 0$, the result (3.4) follows by continuity.

Theorem 3.2 If $A=\left(a_{i j}\right)$ and $B=\left(b_{i j}\right)$ are nonsingular $M$-matrices, then

$$
\begin{aligned}
\tau(A \star B) \geq & \frac{1}{2} \min _{i \neq j}\left\{a_{i i} b_{i i}+a_{j j} b_{j j}-\left[\left(a_{i i} b_{i i}-a_{j j} b_{j j}\right)^{2}\right.\right. \\
& \left.\left.+4 s_{i} s_{j}\left(\sum_{k \neq i} \frac{\left|b_{k i}\right|}{m_{k}}\right)\left(\sum_{l \neq j} \frac{\left|b_{l j}\right|}{m_{l}}\right)\right]^{\frac{1}{2}}\right\} .
\end{aligned}
$$

Proof Obviously, inequality (3.7) holds with equality for $n=1$. Therefore, we assume that $n \geq 2$ and give two cases to prove this problem.

Case 1. Suppose that $C=A \star B$ is irreducible, then $A$ and $B$ are also irreducible. By Lemma 2.2 , there exists a pair $(i, j)$ of positive integers with $i \neq j(1 \leq i, j \leq n)$ such that

$$
\begin{aligned}
\left|\tau(A \star B)-a_{i i} b_{i i}\right|\left|\tau(A \star B)-a_{j j} b_{j j}\right| & \leq\left(s_{i} \sum_{k \neq i} \frac{\left|a_{k i} b_{k i}\right|}{s_{k}}\right)\left(s_{j} \sum_{l \neq j} \frac{\left|a_{l j} b_{l j}\right|}{s_{l}}\right) \\
& \leq\left(s_{i} \sum_{k \neq i} \frac{\left|a_{k i} b_{k i}\right|}{\left|a_{k i}\right| m_{k}}\right)\left(s_{j} \sum_{l \neq j} \frac{\left|a_{l j} b_{l j}\right|}{\left|a_{l j}\right| m_{l}}\right) \\
& =\left(s_{i} \sum_{k \neq i} \frac{\left|b_{k i}\right|}{m_{k}}\right)\left(s_{j} \sum_{l \neq j} \frac{\left|b_{l j}\right|}{m_{l}}\right) .
\end{aligned}
$$

From inequality (3.8) and $0 \leq \tau(A \star B) \leq a_{i i} b_{i i}$ (see [8]), for any $i \in N$, we have

$$
\left(\tau(A \star B)-a_{i i} b_{i i}\right)\left(\tau(A \star B)-a_{j j} b_{j j}\right) \leq\left(s_{i} \sum_{k \neq i} \frac{\left|b_{k i}\right|}{m_{k}}\right)\left(s_{j} \sum_{l \neq j} \frac{\left|b_{l j}\right|}{m_{l}}\right) .
$$

Thus, by solving quadratic inequality (3.9), we have that

$$
\tau(A \star B) \geq \frac{1}{2} \min _{i \neq j}\left\{a_{i i} b_{i i}+a_{j j} b_{j j}-\left[\left(a_{i i} b_{i i}-a_{j j} b_{j j}\right)^{2}+4 s_{i} s_{j}\left(\sum_{k \neq i} \frac{\left|b_{k i}\right|}{m_{k}}\right)\left(\sum_{l \neq j} \frac{\left|b_{l j}\right|}{m_{l}}\right)\right]^{\frac{1}{2}}\right\}
$$

i.e., conclusion (3.7) holds.

Case 2. Similarly, if $C=A \star B$ is reducible. It is well known that a matrix in $Z_{n}$ is a nonsingular $M$-matrix if and only if all its leading principal minors are positive (see Condition (E17) of Theorem 6.2 .3 of [8]). If we denote by $P=\left(p_{i j}\right)$ the $n \times n$ permutation matrix $\left(p_{i j}\right)$ with

$$
p_{12}=p_{23}=\cdots=p_{n-1, n}=p_{n, 1}=1
$$


the remaining $p_{i j}$ being zero, then both $A-\varepsilon P$ and $B-\varepsilon P$ are irreducible nonsingular $M$-matrices for any sufficiently small positive real number $\varepsilon$ such that all the leading principal minors of both $A-\varepsilon P$ and $B-\varepsilon P$ are positive. Now we substitute $A-\varepsilon P$ and $B-\varepsilon P$ for $A$ and $B$, respectively, in the previous Case 1 , and then letting $\varepsilon \rightarrow 0$, the result (3.7) follows by continuity.

Remark 3.1 Similarly, by solving quadratic inequality (3.9) and the same proof as Theorem 3.2, one can also obtain an upper bound on the $\tau(A \star B)$ :

$$
\begin{aligned}
\tau(A \star B) \leq & \frac{1}{2} \max _{i \neq j}\left\{a_{i i} b_{i i}+a_{i j} b_{j j}\right. \\
& \left.+\left[\left(a_{i i} b_{i i}-a_{j j} b_{j j}\right)^{2}+4 s_{i} s_{j}\left(\sum_{k \neq i} \frac{\left|b_{k i}\right|}{m_{k}}\right)\left(\sum_{l \neq j} \frac{\left|b_{l j}\right|}{m_{l}}\right)\right]^{\frac{1}{2}}\right\} .
\end{aligned}
$$

Remark 3.2 Next, we give a comparison between inequality (3.4) and inequality (3.7). Without loss of generality, for $i \neq j$, we assume that

$$
a_{i i} b_{i i}-s_{i} \sum_{k \neq i} \frac{\left|b_{k i}\right|}{m_{k}} \leq a_{i j} b_{j j}-s_{j} \sum_{l \neq j} \frac{\left|b_{l j}\right|}{m_{l}}
$$

Thus, we can rewrite (3.10) as

$$
s_{j} \sum_{l \neq j} \frac{\left|b_{l j}\right|}{m_{l}} \leq a_{j j} b_{j j}-a_{i i} b_{i i}+s_{i} \sum_{k \neq i} \frac{\left|b_{k i}\right|}{m_{k}} .
$$

From (3.11), we have that

$$
\begin{aligned}
& \left(a_{i i} b_{i i}-a_{j j} b_{j j}\right)^{2}+4\left(s_{i} \sum_{k \neq i} \frac{\left|b_{k i}\right|}{m_{k}}\right)\left(s_{j} \sum_{l \neq j} \frac{\left|b_{l j}\right|}{m_{l}}\right) \\
& \leq\left(a_{i i} b_{i i}-a_{j j} b_{j j}\right)^{2}+4 s_{i} \sum_{k \neq i} \frac{\left|b_{k i}\right|}{m_{k}}\left(a_{i j} b_{j j}-a_{i i} b_{i i}+s_{i} \sum_{k \neq i} \frac{\left|b_{k i}\right|}{m_{k}}\right) \\
& \leq\left(a_{i i} b_{i i}-a_{j j} b_{j j}\right)^{2}+4 s_{i} \sum_{k \neq i} \frac{\left|b_{k i}\right|}{m_{k}}\left(a_{j j} b_{j j}-a_{i i} b_{i i}\right)+4\left(s_{i} \sum_{k \neq i} \frac{\left|b_{k i}\right|}{m_{k}}\right)^{2} \\
& =\left(a_{i j} b_{i j}-a_{i i} b_{i i}+2 s_{i} \sum_{k \neq i} \frac{\left|b_{k i}\right|}{m_{k}}\right)^{2} .
\end{aligned}
$$

Thus, from (3.7) and the above inequality, we can obtain

$$
\begin{aligned}
\tau(A \star B) & \geq \frac{1}{2} \min _{i \neq j}\left\{a_{i i} b_{i i}+a_{j j} b_{i j}-\left[\left(a_{i i} b_{i i}-a_{i j} b_{j j}\right)^{2}+4 s_{i} s_{j}\left(\sum_{k \neq i} \frac{\left|b_{k i}\right|}{m_{k}}\right)\left(\sum_{l \neq j} \frac{\left|b_{l j}\right|}{m_{l}}\right)\right]^{\frac{1}{2}}\right\} \\
& \geq \min _{i \neq j} \frac{1}{2}\left\{a_{i i} b_{i i}+a_{j j} b_{i j}-a_{j j} b_{j j}+a_{i i} b_{i i}-2 s_{i} \sum_{k \neq i} \frac{\left|b_{k i}\right|}{m_{k}}\right\} \\
& \geq \min _{1 \leq i \leq n}\left\{a_{i i} b_{i i}-s_{i} \sum_{k \neq i} \frac{\left|b_{k i}\right|}{m_{k}}\right\} .
\end{aligned}
$$


Hence, the bound in (3.7) is sharper than the bound in (3.4).

Next, let us consider a simple example.

Example 3.1 [1] Consider two $4 \times 4 M$-matrices

$$
A=\left(a_{i j}\right)=\left(\begin{array}{cccc}
4 & -1 & -1 & -1 \\
-2 & 5 & -1 & -1 \\
0 & -2 & 4 & -1 \\
-1 & -1 & -1 & 4
\end{array}\right), \quad B=\left(b_{i j}\right)=\left(\begin{array}{cccc}
1 & -\frac{1}{2} & 0 & 0 \\
-\frac{1}{2} & 1 & -\frac{1}{2} & 0 \\
0 & -\frac{1}{2} & 1 & -\frac{1}{2} \\
0 & 0 & -\frac{1}{2} & 1
\end{array}\right)
$$

By calculation, we obtain that $\tau(A \star B)=3.2296$. If we apply (3.1), we can get that

$$
\tau(A \star B) \geq \tau(A) \tau(B)=0.1910 .
$$

If we apply (3.2), we have that

$$
\tau(A \star B) \geq \min _{1 \leq i \leq n}\left\{a_{i i} \tau(B)+b_{i i} \tau(A)-\tau(A) \tau(B)\right\}=1.5730
$$

If we apply (3.3), we have

$$
\begin{aligned}
\tau(A \star B) \geq & \max _{1 \leq i \leq n} \frac{1}{2}\left\{a_{i i} b_{i i}+a_{j j} b_{j j}-\left[\left(a_{i i} b_{i i}-a_{j j} b_{j j}\right)^{2}\right.\right. \\
& \left.\left.+4\left(a_{i i}-\tau(A)\right)\left(b_{i i}-\tau(B)\right)\left(a_{j j}-\tau(A)\right)\left(b_{j j}-\tau(B)\right)\right]^{\frac{1}{2}}\right\} \\
= & 1.573 .
\end{aligned}
$$

If we apply (3.4), we have that

$$
\tau(A \star B) \geq \min _{1 \leq i \leq n}\left\{a_{i i} b_{i i}-s_{i} \sum_{j \neq i} \frac{\left|b_{j i}\right|}{m_{j}}\right\}=2.8333
$$

If we apply (3.7), we get that

$$
\begin{aligned}
\tau(A \star B) & \geq \frac{1}{2} \min _{i \neq j}\left\{a_{i i} b_{i i}+a_{j j} b_{j j}-\left[\left(a_{i i} b_{i i}-a_{j j} b_{j j}\right)^{2}+4 s_{i} s_{j}\left(\sum_{k \neq i} \frac{\left|b_{k i}\right|}{m_{k}}\right)\left(\sum_{l \neq j} \frac{\left|b_{l j}\right|}{m_{l}}\right)\right]^{\frac{1}{2}}\right\} \\
& =2.9199 .
\end{aligned}
$$

From the above example, inequality (3.7) is obviously the best one corresponding to inequalities (3.1), (3.2), (3.3) and (3.4). 


\section{Acknowledgements}

This paper is supported by the National Natural Science Foundation of China $(11026085,11101071,11271001,51175443)$

and the Fundamental Research Funds for China Scholarship Council.

Received: 30 May 2013 Accepted: 12 August 2013 Published: 13 September 2013

\section{References}

1. Li, YT, Li, YY, Wang, RW, Wang, YQ: Some new bounds on eigenvalues of the Hadamard product and the Fan product of matrices. Linear Algebra Appl. 432, 536-545 (2010)

2. Liu, QB, Chen, GL: On two inequalities for the Hadamard product and the Fan product of matrices. Linear Algebra Appl. 431, 974-984 (2009)

3. Horn, RA, Johnson, CR: Topics in Matrix Analysis. Cambridge University Press, Cambridge (1985)

4. Fang, MZ: Bounds on the eigenvalues of the Hadamard product and the Fan product of matrices. Linear Algebra Appl. 425, 7-15 (2007)

5. Zhou, D, Chen, G, Wu, G, Zhang, X: On some new bounds for eigenvalues of the Hadamard product and the Fan product of matrices. Linear Algebra Appl. 438, 1415-1426 (2013)

6. Varga, RS: Minimal Gerschgorin sets. Pac. J. Math. 15, 719-729 (1965)

7. Horn, RA, Johnson, CR: Matrix Analysis. Cambridge University Press, New York (1985)

8. Berman, A, Plemmons, RJ: Nonnegative Matrices in the Mathematical Sciences. Academic Press, London (1978)

doi:10.1186/1029-242X-2013-433

Cite this article as: Guo et al.: New inequalities on eigenvalues of the Hadamard product and the Fan product of matrices. Journal of Inequalities and Applications 2013 2013:433.

\section{Submit your manuscript to a SpringerOpen ${ }^{\circ}$ journal and benefit from:}

- Convenient online submission

Rigorous peer review

- Immediate publication on acceptance

Open access: articles freely available online

- High visibility within the field

- Retaining the copyright to your article 